\title{
Mid-IR characterization of substellar companions with CanariCam
}

\author{
V. J. S. Béjar ${ }^{1,2, a}$, B. Gauza ${ }^{1,2}$, R. Rebolo ${ }^{1,2}$, C. Álvarez ${ }^{1,2}$, M. R. Zapatero Osorio ${ }^{3}$, and L. Labadie ${ }^{4}$ \\ 1 Instituto de Astrofísica de Canarias. C / Vía Láctea s/n, E-38200. La Laguna (Tenerife), Spain \\ 2 Departamento de Astrofísica, Universidad de La Laguna, E-38205. La Laguna, Tenerife, Spain \\ 3 Centro de Astrobiología (CSIC-INTA), Ctra. de Ajalvir, km 4, E-28850, Torrejón de Ardoz, Spain \\ ${ }^{4}$ Physikalisches Institut, Universität zu Köln, Zülpicher Str. 77, 50937 Köln, Germany
}

\begin{abstract}
The direct detection of extrasolar planets is the only technique that leads to the most complete characterization of their physical properties. Here, we report on mid-IR observations of five directly imaged substellar companions (DF Tau B, FU Tau B, $\kappa$ And b, GJ $758 \mathrm{~B}$, and GJ $504 \mathrm{~b}$ ) obtained with the Si-2 filter $(8.7 \mu \mathrm{m})$ in the CanariCam instrument mounted on the 10-m Gran Telescopio de Canarias (GTC). We show the detection of FU Tau B, and we set upper limits of 11.5-12.0 mag in the $8.7 \mu \mathrm{m}$ band for the other substellar targets. These data are useful to constrain their effective temperature and the presence of mid-IR flux excesses that may be due to surrounding disks.
\end{abstract}

\section{Introduction}

Since the first direct detection of a planetary-mass companion around the brown dwarf 2MASS J12073932 [1], several low-mass substellar companions have been found. Some examples are FU Tau B, DH Tau B, $\kappa$ And b, GJ 758 B, and GJ 504 b (see [2-6]). The study of these wide companions is fundamental to understand the formation of planetary systems and to describe the physical properties of isolated planetary-mass objects and extrasolar planets discovered by transits and radial velocity techniques, because they have very similar effective temperatures and gravities.

\section{Observations and data analysis}

Mid-IR images of FU Tau B, DH Tau B, $\kappa$ And b, GJ 758 B, and GJ 504 b were obtained using the Si-2 filter $\left(\lambda_{c}=8.7 \mu \mathrm{m}, \delta \lambda=1.1 \mu \mathrm{m}\right)$ of the CanariCam instrument on the GTC on September 21, 30, October 3, 2013 and March 3, 2014. Observations were done in queue mode and using the standard chopping and nodding technique in the mid-IR. Weather conditions were clear and the amount of precipitable water vapor ranged from 5 to $15 \mathrm{~mm}$. CanariCam data are composed of multi-extension FITS format images of different chop and nod positions. Individual exposures of $26 \mathrm{~ms}$ are co-added before being saved every 1.6 or $6 \mathrm{~s}$. Total on-source integration time was 21-22 min per Observing Block (OB). One OB was obtained for FU Tau and GJ 504, two OBs for DH Tau and three OBs for $\kappa$ And. Data were reduced using a CL script of IRAF developed by our group. This processing software includes sky and radiative offset subtraction, and proper shift-and-add of the images. Because the chop throw angle was 8 arcsec, the source was always present within the field of view on both the on- and off-source images. During the process of reduction the off-source fluxes of the target were inverted, aligned and co-added to the on-source flux. The CanariCam final images of these substellar companions can be seen in Figure 1. Their mid-IR magnitudes and near- to mid-IR colors are shown in Table 1.

a e-mail: vbejar@iac.es

This is an Open Access article distributed under the terms of the Creative Commons Attribution License 4.0, which permits unrestricted use, distribution, and reproduction in any medium, provided the original work is properly cited. 

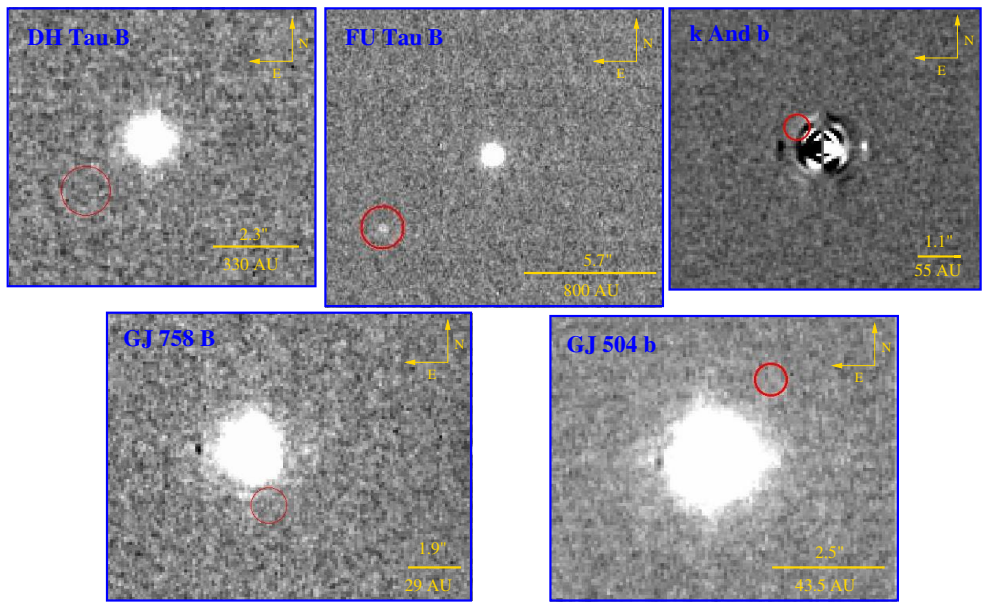

Fig. 1. CanariCam $8.7 \mu \mathrm{m}$ images of DH Tau, FU Tau, $\kappa$ And, GJ 758 and GJ 504 systems. The red circle marks the position of the companions. A rotated and self-subtracted PSF image of $\kappa$ And is shown.

Table 1. CanariCam mid-IR photometry of companions.

\begin{tabular}{lccc}
\hline Name & {$[8.7](\mathrm{mJy})$} & {$[8.7](\mathrm{mag})$} & $J$-[8.7] $(\mathrm{mag})$ \\
\hline DH Tau B & $>1.3$ & $>11.5$ & $<4.2$ \\
FU Tau B $^{a}$ & $4 \pm 0.5$ & $10.3 \pm 0.1$ & $4.70 \pm 0.14$ \\
$\kappa$ And b & $>0.8$ & $>12.0$ & $<4.8$ \\
GJ 758 B & $>1.0$ & $>11.8$ & $<6.7$ \\
GJ 504 b & $>2.0$ & $>11.0$ & $<8.8$ \\
\hline
\end{tabular}

a. Calibration was done by interpolation of the primary Wise and Spitzer photometry given in [3]

\section{Results}

- The detection of the young ( 1Myr) FU Tau B $([8.7]=10.3 \pm 0.1)$ suggests the existence of mid-IR flux excesses $(J-[8.7]=4.70 \pm 0.14$, compared to $J-[8.0]=2.0-2.4$ for late M dwarfs [7]) that may be due to the presence of a disk. We measure a separation of 5.74 " \pm 0.03 between FU Tau A and B $(5.56 " \pm 0.05$ arcsec is expected for a background star), in agreement with the values of 5.61-5.72" found by [3], confirming that both objects share a common proper motion and belong to Taurus.

- The non detection of DH Tau B and $\kappa$ And b is consistent with their L spectral types and indicates that these young ( $\sim 1$ and $30 \mathrm{Myr}$, respectively) substellar companions have no mid-IR excesses.

- The upper limits imposed to GJ 758 B and GJ 504 b allow us to determine that the former has an spectral type earlier than Y dwarf (probably it is a late T) and the later is earlier than Y1. Hence, their effective temperatures are estimated to be higher than $\sim 500 \mathrm{~K}$ and $\sim 350 \mathrm{~K}$, respectively.

\section{References}

1. Chauvin et al., A\&A 425, (2004) L29

2. Itoh, Y., et al., ApJ 620, (2005) 984

3. Luhman et al., ApJ 691, (2009) 1265

4. Thalmann et al., ApJ 707, (2009) L123

5. Carson, J. et al., ApJ 763, (2013) L32

6. Kuzuhara et al. ApJ 774, (2013 11

7. Patten et al. ApJ 651, (2006) 502 